\title{
Revisión teórica de modelos de gestión logístico para el sector artesanal Macanero Gualaceo- Ecuador
}

Theoretical review of logistics management models for the Macanero artisan sector in Gualaceo- Ecuador

Mónica Briggith Rosales Namicela. ${ }^{1}$

Recibido: 07-07-2021 / Revisado: 21-07-2021 /Aceptado: 04-08-2021/ Publicado: 05-09-2021

\begin{abstract}
.
DOI: https://doi.org/10.33262/concienciadigital.v4i3.2.1842

Introduction: The globalizing processes accompanied by technological and communicational advances to which organizations are forced to incorporate to sustain themselves in the market, has made the conditions of the business environment become complex, mainly affecting activities such as artisanal whose characteristics are high vulnerability but that require incorporating logistical processes to sustain the activity. Objectives: Faced with this reality, the need arises to determine a logistics management model of the supply chain for the Macanero artisan textile activity of the GualaceoEcuador Canton. Methodology: This research is framed within a bibliographic design of a documentary type at a historical level to understand the evolution of concepts, practices and models for the implementation of logistics management in organizations. Results: There have been several contributions made on this subject, Velásquez (2003) developed various tools and strategies to promote a change from some SMEs in a natural state to innovative small and medium-sized companies, so that they fit into the current business world but adjusted to their context and their capacities without losing the horizon of being part of organizations of the social and solidarity economy. Conclusions: There are problems in the logistics performance of SMEs in Latin America mainly structurally,
\end{abstract}

\footnotetext{
${ }^{1}$ Universidad Internacional Iberoamericana- México(UNINI), Aspirante a doctor en Derecho Económico y de la Empresa, Campeche-México, mrosalesn@ ucacue.edu.ec, https://orcid.org/0000-0002-3240-1146
} 
alternatives are proposed that allow these production structures to overcome the limitations through an efficient, efficient and effective logistics administration, incorporating the proper management of the supply chain, technological innovation and permanent training.

Keywords: Macanero artisanal textile sector, logistics management, social and solidarity economy, supply chain

\section{Resumen.}

Introducción: Los procesos globalizadores acompañados de avances tecnológicos y comunicacionales al que se ven obligados a incorporar las organizaciones para sostenerse en el mercado, ha hecho que las condiciones del entorno empresarial se vuelvan complejas, afectando principalmente a actividades como la artesanal cuyas características son de elevada vulnerabilidad pero que requieren incorporar procesos logísticos para el sostenimiento de la actividad. Objetivos: Ante esta realidad surge la necesidad de determinar un modelo de gestión logístico de la cadena de suministros para la actividad textil artesanal macanera del Cantón Gualaceo-Ecuador. Metodología: La presente investigación se enmarca dentro de un diseño bibliográfico de tipo documental a nivel histórico para entender la evolución de conceptos, prácticas y modelos para la implementación de la gestión logística en las organizaciones. Resultados: Han sido varios los aportes realizadas sobre esta temática, Velásquez (2003) desarrolló varias herramientas y estrategias para propiciar un cambio de unas pymes en estado natural a pequeñas y medianas empresas innovadora, para que se acoplen al mundo empresarial actual pero ajustadas a su contexto y a sus capacidades sin perder el horizonte de ser parte de organizaciones de la economía social y solidaria. Conclusiones: Existen problemas en el desempeño logístico de la PYMES en América Latina principalmente en lo estructural, se proponen alternativas que permitan a estas estructuras productivas superar las limitaciones mediante una administración logística eficaz, eficiente y efectiva, incorporando el manejo adecuado de la cadena de suministros, la innovación tecnológica y la capacitación permanente.

Palabras claves: Sector textil artesanal macanero, gestión logística, economía social y solidaria, cadena de suministros.

\section{Introducción.}

Los esquemas globalizadores del mundo contemporáneo en los que se desenvuelve las actividades de las micro, pequeñas y medianas empresas, han generado: competencias inhumanas, tratamientos tributarios y arancelarios insostenibles; así también, los avances vertiginosos del desarrollo tecnológico y las comunicaciones a las que sólo pueden acceder las grandes empresas, las políticas de control de precios en los mercados, entre otros factores; han hecho que cada vez las condiciones del mercado se vuelvan difíciles 
de sostener principalmente, para actividades de tipo artesanal y familiar en las que el aporte del ser humano es lo fundamental y en donde quienes se encargan de esta labor son personas en condiciones de vulnerabilidad.

Frente a este panorama sombrío las micro, pequeñas y medianas empresas (MIPYMES), deben hacer frente a esta realidad, incorporando procesos de mejora continua si lo que buscan es mantenerse en el mercado y explorar nuevos espacios en el contexto nacional e internacional; para ello se requiere, introducir avances tecnológicos, comunicaciones e innovación, incorporar criterios de calidad en sus procesos, aplicar esquemas de integración horizontal y vertical, es decir hacer uso de criterios de logística para seguir fortaleciendo su capacidad productiva, generadora de empleo y desarrollo para la localidad en donde se desenvuelven.

La logística se ha convertido en las últimas décadas en un elemento clave en la gestión de los negocios y porque principalmente según (Kirby, Carlos; Brosa, 2011), plantean que esta permite promover el crecimiento del sector privado y alcanzar un mayor desarrollo de un país o territorio por el "efecto de tracción" (p. 26) que la misma genera; esto significa que no solo se fortalece un sector de la economía sino que a través de los encadenamientos locales, generar un desarrollo de una localidad, región y país. Los autores además sostienen que el tener una logística eficiente dentro de las empresas y principalmente para las Pymes les permite competir con éxito en los nuevos mercados globales que se han ido desarrollando.

Las empresas $\mathrm{u}$ organizaciones artesanales para ser sostenibles en mercados globales, necesitan una adecuada gestión logística en función de su propio escenario de desempeño y de su propia realidad en las diferentes etapas de producción y comercialización, que les permita alcanzar mayor competitividad, sostenibilidad y eficiencia en el proceso.

A partir de la necesidad de preservar la actividad macanera para los actores locales por que representa una actividad identitaria, así también es la principal fuente de ingresos de los habitantes de los sectores de Bullcay y Bulzhun del Cantón Gualaceo, de la Provincia del Azuay en el Ecuador; para los entes gubernamentales ha sido una preocupación permanente la situación que atraviesa la actividad macanera, debido a que el desarrollo del proceso productivo con el uso de la técnica ikat es un representante de la cultura y la historia del territorio y esta ha sido declarada en el año 2015 como Patrimonio Inmaterial del Ecuador (Velasco, 2015).

Las concepciones actuales respecto al desarrollo de los territorios está generando nuevas visiones de cómo a través del fomento de actividades y potencialidades del territorio, se promueve un desarrollo sostenible y sustentable mediante las propuestas o concepciones del desarrollo endógeno, que según lo plantea (Vásquez Barquero, 2007), se pueden nombrar de distintas maneras pero siempre buscando alcanzar el desarrollo con un enfoque del ser humano, es decir lograr una mejora en la calidad de vida del mismo.

A pesar de todos los atributos y bondades que posee la actividad artesanal, carece de un reconocimiento justo en el mercado del valor agregado que representa: el tiempo de 
dedicación, el esfuerzo que demanda la actividad y del valor intercultural y trasmisión intergeneracional que significa la actividad textil artesanal. Por este motivo, se ha venido observando con el paso de los años el envejecimiento de los productores artesanales macaneros, la población joven de la localidad no se siente motivada a replicar dicha actividad debido a que los ingresos que perciben los artesanos, no les permite atender sus necesidades básicas y peor aún alcanzar una adecuada calidad de vida.

Ante esta realidad crítica del sector, surge la necesidad de atender el problema: ¿Cómo generar un modelo de gestión logístico para la actividad artesanal macana del Cantón Gualaceo, que le permita la sostenibilidad y posicionar los productos en el mercado nacional e internacional a un justo precio?

A partir de la pregunta de investigación surgen nuevas interrogantes que son importantes de considerar:

1. ¿Existen modelos de gestión logísticos desarrollados para empresas textiles artesanales en otros contextos que han permitido su incursión en los mercados locales e internacionales?,

2. ¿Cuáles son las condiciones de abastecimiento, producción y comercialización que poseen los artesanos macaneros, que limitan su incursión en el mercado nacional e internacional?,

3. ¿Qué políticas, instituciones y legislación gubernamental, son necesarias de considerar para promover y proteger al sector textil artesanal, como un sector estratégico para la generación de empleo y alcanzar el buen vivir de la población vulnerable de la localidad?,

4. ¿Qué variables e indicadores son estratégicos de incorporar en el modelo de gestión logístico, que permita alcanzar la sostenibilidad y lograr posicionar los productos de forma efectiva en los mercados nacionales e internacionales?

A partir de la problemática propuesta y del interés que ha despertado la presente investigación, se ha planteado como objetivo general de la investigación:

Determinar un modelo de gestión logístico para la actividad textil artesanal de la macana para los procesos de abastecimiento, producción, comercialización y distribución de los productos elaborados.

Para alcanzar este objetivo será necesario, sistematizar algunos pasos que permitan; alcanzar los fines de la investigación, por ello se han propuesto como objetivos específicos para su consecución:

1. Analizar teorías, conceptos y el estado del arte de modelos de gestión logístico desarrollados para MIPYMES y organizaciones textiles artesanales considerando, aquellos factores o variables que inciden estratégicamente en la valoración y posicionamiento de la actividad en el mercado local, nacional e internacional.

2. Desarrollar un diagnóstico de los procesos actuales de abastecimiento, producción, comercialización, promoción e innovación tecnológica y de 
comunicaciones que posee el sector textil artesanal macanero del Cantón Gualaceo en el Ecuador.

3. Proponer un Modelo de Gestión Logístico para el sector textil artesanal de la Macana del sector de Bullcay del Cantón Gualaceo que responda a las características propias de la actividad.

\section{Desarrollo}

Para el tratamiento del tema propuesto, se hace necesario recorrer algunas teorías y modelos que se han desarrollado en el transcurso del tiempo y que han sido puestas al servicio de un sector importante de la economía de una localidad o país como es el sector textil, también se han abierto puertas al debate y generación de nuevos planteamientos y propuestas para aportar con nuevos conocimientos y propuestas que propendan a la sostenibilidad de la actividad artesanal, que es un referente de la identidad cultural, ancestral y de tradiciones de una localidad, además por ser un sector importante del desarrollo de muchos pueblos y localidades tradicionalmente artesanales. Para el caso que nos convoca, es aún más necesario generar procesos de discusión y alternativas que permitan atender a sectores vulnerables de la sociedad como es el caso de los actores de la economía popular y solidaria, que en su mayoría son mujeres excluidas del mercado laboral tradicional por ser mujeres campesinas, además por efectos de la migración se han convertido en pilares fundamentales para la sostenibilidad de sus familias.

\section{La economía Social y Solidaria y el Desarrollo Local}

Ante las graves secuelas que ha dejado el modelo capitalista que por mencionar algunas tenemos pobreza, marginación, exclusión, inequidades, entre otras; surge una nueva propuesta de actividad económica que no puede ser considerada como empresa privada porque no busca el lucro o la ganancia como razón final de su actividad y tampoco de la empresa pública por no valerse de recursos públicos para la generación de un bien o servicio; esta estructura organizativa ha recibido desde su reconocimiento como actividad económico-productiva diferentes acepciones, tales como economía del pueblo, economía de la solidaridad, economía social, entre otras. La economía social a diferencia de lo que expresa la economía concebida desde su acepción como ciencia, incorpora elementos sociales y estructuras desarrolladas con democracia y una especie de empresariado colectivo (Lévesque \& Mendell, 2002).

Esta economía no podemos aseverar que es nueva, surgió en el siglo XIX, en donde no sólo se hablaba del surgimiento de estructuras cooperativas formadas por los trabajadores, era una respuesta a la "economía hecha por los economistas; este concepto aparece en la economía occidental bajo dos enfoques; el enfoque social-cristiano (solidarista), que buscaba paliar los grandes costos humanos, económicos y sociales producto de la revolución industrial y la socialista que trataba los procesos de autogestión de la clase trabajadora (Bastidas-Delgado y Richer, 2001). En la actualidad ante las crisis permanentes que afronta el modelo económico capitalista vigente y las graves consecuencias generadas como hambre, pobreza, marginación, exclusión, entre otros; la 
economía social ha sido reconocida en muchos países y regiones del planeta no sólo por las personas sino también por el estado como un sector económico más, integrado por personas asociadas cuya producción y gestión busca satisfacer las necesidades de sus miembros y de la sociedad en general. Ibdem

Las organizaciones que son parte de la economía social y se han fundado bajo principios de ayuda mutua y solidaridad, están tomando una fuerza sin precedentes en el concierto local y mundial, están surgiendo nuevas estructuras distintas a las tradicionales que, basadas en valores como la democracia, inclusión, justicia social, equidad, están cambiando los paradigmas en la búsqueda de soluciones al desempleo, hambre, pobreza y marginación que el mercado, el Estado y el modelo económico vigente, no han sido capaces de resolver. Estos procesos se han venido dando en todo el planeta y es de reconocer que en américa latina en las últimas décadas los gobiernos han reconocido dentro de su legislación políticas e instancias, orientadas a apoyar y fomentar a este nuevo sector (Bastidas-Delgado y Richer, 2001).

La concepción de economía social y su incidencia en el desarrollo de los países, ha sido un proceso de construcción en permanente transformación y desde los diferentes estamentos de la sociedad, Estado y comunidad científica, se la ha venido nombrando de diferentes formas social, solidaria, popular; pero lo común de todas ellas es que se prioriza el trabajo y no al capital. Se puede concluir sosteniendo que la economía social solidaria acoge a aquellas organizaciones, asociaciones, cooperativas que no persiguen fines de lucro, basadas en valores y principios cuyos beneficios serán distribuidos de manera equitativa y en beneficio de la organización en su conjunto (Bastidas-Delgado y Richer, 2001).

Otro aspecto importante al cual aporta de manera significativa este sector es al desarrollo local, se han planteado algunas propuestas actuales desde una visión más integral del desarrollo a aquellos elementos conocidos con satisfactores, que según Max-Neef propone una matriz en la cual se incorpora no solo elementos materiales sino también aspectos del hombre entendidos desde la subjetividad de la valoración que cada persona da a su desarrollo personal en su "ser, hacer, tener y estar" (Max-Neef et al., 2010).

Por lo expresado (Max-Neef et al., 2010), hace referencia a la teoría del desarrollo desde la óptica humana "aclara las relaciones socioculturales de....correspondencia entre las necesidades y los satisfactores" (p.34) esto con el fin de que los bienes y servicios generados en cada sociedad permitan atender de manera adecuada dichas necesidades, vistas como carencias por esa sociedad y que son fundamentales obtener para alcanzar el "Buen Vivir". Los satisfactores no solo entendidos como bienes tangibles sino también “...puede incluir formas de organización, estructuras políticas, prácticas sociales, ....valores, normas, espacios, contextos, comportamiento y actitudes" (p.38), elementos en permanente consolidación y evolución de acuerdo al momento histórico que enfrenta dicha sociedad.

Alcanzar el desarrollo desde la mirada de Sen se logra a través del fortalecimiento de las libertades. (Sen, 1998) sostiene que son procesos de ampliación de las libertades reales 
que deben gozar los individuos; este desarrollo no debe entenderse como meramente económico, se plantea como elementos determinantes para su consecución, los derechos políticos y humanos mediante una participación activa en la construcción de acciones y políticas gubernamentales, por tanto el desarrollo exige eliminar las principales fuentes de carencias de libertades como: pobreza, tiranía, falta de oportunidades económicas, ausencia de servicios públicos y la intolerancia o el exceso de intervención de Estados represivos.

A más de concebir el desarrollo como tal se ha agregado una nueva visión desde la mirada local de participación de todos los actores tanto de lo público como son los gobiernos locales, como desde lo privado es decir desde los emprendimientos con aprovechamiento de las potencialidades locales.

Bajo esta visión de un desarrollo económico y social, la acción preponderante de las instancias gubernamentales locales, son ejes claves impulsando las iniciativas y potencialidades locales para que actuando de manera efectiva, eficiente y coordinada con los actores políticos, económicos y sociales generen un desarrollo productivo sostenible.

\section{La mujer y su aporte al desarrollo local}

En la sociedad actual no se puede concebir que se da una neutralidad del género en temas de desarrollo local, debido a que se evidencia en muchos aspectos e instancias de la vida pública, relaciones laborales y de poder con fuertes desigualdades entre hombres y mujeres; el género es un determinante de los procesos de desarrollo local en los territorios, es un elemento transversal que modula a los otros aspectos del desarrollo local; por ello es necesario que las políticas públicas no sólo este dirigidas a atender temas referentes a la condición de la mujer sino a cambiar las estructuras de subordinación y discriminación existentes en todos los espacios de acción dentro de la sociedad (Massolo, 2007).

Es una necesidad estratégica para promover el desarrollo local, generar profundas transformaciones en las relaciones sociales, para que la mujer disfrute de una auténtica libertad, autoestima y empoderamiento en función al contexto económico social y cultural en donde se desenvuelve; esto se verá reflejado en una mejor convivencia, reducción de los niveles de violencia y exclusión a los cuales se ven inmersa las mujeres. Es uno de los principales retos para el desarrollo local, elevar la posición e interés de la mujer para contar con un ser colectivo y constructivo del desarrollo con una auténtica participación de la mujer en la vida sociopolítica de la localidad y alcanzar mejorar la calidad de vida de todos y todas. Ibdem

En función de lo expresado, es necesario promover un verdadero desarrollo local inclusivo con equidad de género, interviniendo en problemáticas de:

- Graves desigualdades en la distribución de bienes y servicios, producto de la discriminación, incapacidad de las mujeres para decidir sobre su cuerpo, nulo acceso a la propiedad de los bienes 
- Desigualdad en la valoración del trabajo entre hombres y mujeres e inequitativo aporte del trabajo de las mismas al patrimonio de sus familias

- Políticas públicas diferenciadas entre hombres y mujeres, que inciden en la poca valoración de la misma por parte de la sociedad en los ámbitos sociales, económicos y reproductivos, lo que la invisibiliza como ser con identidad propia con intereses, ideas y puntos de vista propios (Massolo, 2007)

(Massolo, 2006), aduce que en lo local si se plantean programas o proyecto, estos deben ser desarrollados desde una perspectiva de género, caso contrario partirían de datos sesgados que no aportarían a generar acciones desde lo local con equidad de género.

Bajo estos análisis, la legislación nacional ecuatoriana establece abordar los temas locales con perspectivas de género en todos los estamentos públicos, en los procesos de construcción y gestión local; es necesarios implementar políticas y acciones con un enfoque de género que permitan superar los estereotipos vigentes en la sociedad y promover procesos de desarrollo más inclusivos y participativos de la mujer (Consejo Nacional para la igualdad de Género, 2018).

En los tiempos actuales, en que las mujeres son partícipes en la construcción de acciones y políticas públicas, es aún mal vista por la sociedad, los espacios de acción local participando en política de la comunidad y en donde se generan los tejidos sociales, ha sido el espacio mejor valorado, pero aún sigue siendo insuficiente su reconocimiento y valoración.

Debido a los problemas de inequidades existentes en la sociedad, las mujeres desarrollan proyecto, emprendimientos que generalmente se enmarcan dentro de actividades de tipo informal que le permiten generar ingresos de pobreza para atender las necesidades del grupo familiar. Los procesos de empoderamiento son fundamentales en la sociedad actual, para que las mujeres puedan tener espacios de control y poder sobre sus vidas y hacer usos de sus derechos para construir una vida más digna y desterrar de su estructura mental una condición de discriminación y desigualdad en la que se ha desarrollado por siglos. La participación de la mujer en el desarrollo local es un derecho como ciudadana a gozar de iguales oportunidades como los hombres. Ibdem

\section{La actividad textil artesanal y su incidencia en el desarrollo local}

El sector textil artesanal representa para una localidad una actividad económica importante, debido a que representa la identidad cultural e histórica que distingue a los pueblos ancestrales, (Jha \& Nath, n.d.). Esta actividad en su gran mayoría presenta como debilidad bajos procesos de integración, lo que la hace vulnerable ante los procesos de integración y desarrollo que poseen las grandes multinacionales. La actividad artesanal, busca sostenerse en los mercados mediante estrategias aisladas e individualizadas por parte de los artesanas y escasas políticas públicas de apoyo a las mismas (Rivera Mateos \& Hernández Rojas, 2019). 
La actividad artesanal se ha convertido en los tiempos actuales en un elemento esencial, por representar aspectos identitarios y culturales de un territorio en temas de oferta turística (Gustami et al., 2014), (Jha \& Nath, 2015), de países en vías de desarrollo como de países desarrollados (Richards, 2013) (Richards, 2005), (Maruya et al., 2015), (Gazimagomedov, 2017). La actividad artesanal, ha tenido un reconocimiento por la comunidad internacional por su valor patrimonial y por su aporte al desarrollo de muchos pueblos ancestrales en distintas partes del mundo; para américa latina esta actividad además se ha convertido en un factor de desarrollo importante por representar el sustento socio-económico y una forma de vida de más de la cuarta parte de la población latinoamericana; para territorios con grandes arraigos artesanales, las políticas públicas en algo han permitido generar acciones de protección y fomento debido al reconocimiento mundial que en los últimos años ha tenido esta actividad principalmente como un factor de fomento turístico. (Benítez Aranda, 2009), (Cruz et al., 2008), (Arruda et al., 2013), (Fernández, 2015).

La actividad artesanal en los tiempos actuales, se ha convertido en un insumo impresindicible que aporta valor a los destinos turísticos de los territorios y le agregan un diferencial a dicha localidad para atraer a los distintos tipos de clientes motivados por los aspectos culturales; este producto es un elemento que vuelve atractivo y genera un factor diferenciador de la localidad, que puede ser un determinante para la visita sea esta por experiencias vivenciales, compras de souvenirs o apreciar los procesos artesanalesproductivos. La actividad turística según estudios recientes, han determinado que dicha actividad generar sinergias y encadenamientos que promueven tanto las actividades artesanales de la localidad, empoderando a la mujer rural quien es portadora del conocmiento artesanal ancestral e incidiendo en el desarrollo local; así también esta actividad promueve la generación de empleo del territorio y genera procesos de inclusión de la población que vive en condiciones de marginación o exclusión (Mello \& Ceretta, 2015), (Rodrigues, B., De Sousa, R., Vasconcelo, 2014). (Ashley et al., 2001)

Según estudios desarrollados sobre el sector textil de micro, pequeñas y medianas empresas, han evidenciado los graves problemas multidimensionales que enfrentan como: un nivel bajo de educación de los empresarios, reducido acceso a tecnología e innovación , a la información y comunicación, escaso acceso al financiamiento entre otros; lo que da como resultado un incipiente nivel de competitividad; por ello, se vuelve la actividad cada vez más vulnerable a las exigencias de mercados altamente competitivos y demandantes de productos de elevada calidad y a bajo costo.

Aspilcueta-Barbachan (2000), Castillo (2005), citados por (Larios, 2017), plantean, luego de analizar al sector de las micro, pequeñas y medianas empresas respecto a: los esquemas actuales operativos, los administrativos y, los procesos de financiación con que cuentan para desarrollar sus actividades; que los mismos enfrentan un escenario sombrío que les exige, romper paradigmas a partir de entender su funcionamiento, afrontar los nuevos retos del mundo globalizado y gestionar conjuntamente con los actores gubernamentales, políticas locales participativas y mundiales que promuevan la actividad reconociendo en las misma su potencial como generador de empleo, actor clave desde lo local para mejorar 
la calidad de vida de la población vulnerable y alcanzar por los encadenamientos que generen un desarrollo local sostenible y sustentable.

\section{La logística en la administración}

Es necesario conceptualizar y entender a la logística en el campo de la administración; para ello se parte de definir a la logística según (SRINIVASAN, M.; MUKHERJEE, D.; GAUR, 2008), como un método ejecutado de forma holística. Las actividades que sirven de apoyo al proceso, deben ser ejecutadas de manera integral, efectiva y eficientemente, desde la decisión de adquirir los insumos necesarios para el proceso, hasta la distribución de los bienes o servicios incluyendo en la actualidad el servicio posventa.

Actualmente, el enfoque de la logística no sólo se centra en la gestión óptima de la cadena de suministros y procesos que le permiten atender los requerimientos de los clientes, incorporando un valor agregado y a un costo adecuado; es necesario incorporar otras variables claves por los problemas medioambientales que enfrenta el mundo moderno; organismos como la Organización de la Naciones Unidas (ONU) (2010) citado por (Ramírez Meneses et al., 2016), sostiene que la administración de las cadenas de suministros de las empresas deben incorporar a más de factores que permiten alcanzar una eficiencia económica, también administrar eficientemente los impactos ambientales y sociales, esto representa aplicar procesos de "logística inversa" (Ramírez Meneses et al., 2016, p. 52), se refiere a atender el mercado actual sin afectar a las generaciones futuras.

Se podría por tanto plantear que, la logística es un proceso sistemático de tareas o actividades, llevadas a cabo en cada una de las áreas o departamentos de la organización y que le permitirán, alcanzar las metas propuestas según la estrategia adoptada por la misma pero, considerando procesos sostenibles y mercados potenciales actuales y futuros que la empresa pretende atender.

\section{Liderazgo Estratégico}

Los sistemas administrativos surgieron y se mantuvieron en el pasado de forma natural ya sea por una necesidad o por las propias experiencias de vida; sin embargo, en los momentos actuales donde las empresas que inicialmente era el dueño quien asumía la responsabilidad de mantener su empresa a flote, se enfrenta actualmente a un mercado globalizado bien sea, porque ha decidido incursionar en otros mercados o porque, se ha visto obligado dentro de su mercado local a enfrentar a grandes empresas. Por ello, se debe repensar el concepto de líder; Robbins (1999) citado (Escandon-Barbosa \& HurtadoAyala, 2016) plantean que, un líder es quien genera determinada influencia positiva a través de procesos comunicacionales interactivos, en equipos de personas que conforman conglomerados sociales que persiguen un objetivo común.

En el análisis de los aspectos que destacan de un líder, se han ido construyendo diversas teorías en torno a los rasgos que distinguen a un líder y sobre el comportamiento que desarrolla el líder. La teoría de rasgos de Stogdill (1948) y Ghiselli (1970), citados por 
(Escandon-Barbosa \& Hurtado-Ayala, 2016), determinan que un líder posee rasgos innatos como: su capacidad de reacción ante conflictos de forma oportuna y acertada, proactivos, sociable, inteligentes, íntegros, capaces, cautos; que saben responder de manera oportuna y acertada ante conflictos, haciendo que los miembros de la organización lo sigan para alcanzar metas. La teoría en base al comportamiento de Lewin, Lippit y White (1939) citado por el mismo autor, analiza el liderazgo en base a la conducta y su relación con el liderazgo efectivo (p.139).

Para actuar en esta realidad compleja y de cambios acelerados de la actualidad, se debe desarrollar esquemas de delegación de funciones y responsabilidades, acompañados de acciones de profesionalización de los departamentos para aumentar la eficiencia empresarial. Los procesos administrativos y gerenciales en la actualidad se deben aprovechar de forma más efectiva, entender que por el conocimiento adquirido por la gerencia durante varios años, se encargue exclusivamente de los procesos de planificación del futuro de las compañías, buscar las estrategias más idóneas que le proporcionen una rentabilidad, permanencia y competitividad adecuada.

Otro factor necesario de clarificar es entender dentro de la administración, ¿qué constituyen las organizaciones?; según Sahid Castaño (1998) citado por (Velásquez, 2003), plantea en su análisis de este concepto como un conjunto de agentes que requieren de recursos económicos necesarios para el desarrollo de una actividad productiva y, que a través de su acción sobre estos e incorporando un valor agregado bien sea, de tipo manual o herramientas tecnológicas y mediante procesos de comercialización, alcanzar sus expectativas y satisfacer las necesidades de sus clientes.

Pero las organizaciones en las últimas décadas por la necesidad de sostenerse, se han visto forzadas a adaptarse a un medio globalizada que cambia vertiginosamente en todos los ámbitos; por ello las organizaciones han tenido que buscar mecanismos de especialización de sus áreas o departamentos, pero que deben ser visualizados como un sistema íntimamente relacionado o integrado. Partiendo de estos procesos se ha desarrollado algunos modelos de gestión para cada área o subsistema.

(Velásquez, 2003), desarrolló un modelo para cinco de las áreas o subsistemas que según el autor, son claves para el éxito de la organización; así plantean para el subsistema de dirección y gestión, las funciones básicas es el planteamiento de:

- "Misión, visión, valores y objetivos

- Los principios administrativos

- La gestión de recursos

- Comunicación e información

- La estructura y los procesos" (70-71)

Los procesos organizativos de las empresas en la actualidad, deben ajustarse a una visión integral (holística), para desarrollar una planeación estratégica que le permita, responder a mercados más exigentes y competitivos, cargados de incertidumbre y poder adaptarse a dichas realidades. 
Así también se plantea una estrategia para el subsistema de operaciones, entendiendo que para esta área la variable de mayor incidencia es la demanda que inciden sobre elementos como: los flujos de efectivo, el retorno de la inversión entre otros; para el subsistema de operaciones la influencia de la gestión estratégica es un factor clave que depende de decisiones tomadas a nivel gerencial y de cómo se encuentra la estructura organizacional para que el resto de procesos fluyan. El desempeño y éxito de esta área dependerá de las estrategias aplicadas, de la estructura establecida y de un correcto proceso gerencial de la compañía.

Otro subsistema clave según estudios es el factor logístico que está determinado en base al cliente; lo que toda organización busca es satisfacerlo de manera eficiente; para alcanzar este objetivo es necesario alcanzar una administración estratégica de toda la cadena de abastecimiento. Mientras exista entre ellos una adecuada integración, comunicación y sinergia, mejores resultados se podrán alcanzar (Fred, 2009).

Un modelo para ser efectivo, demanda un proceso de adaptación según la estructura, y necesidades de cada empresa u organización; el modelo propuesto por (Velásquez, 2003), plantea que un esquema estratégico debe integrar los objetivos individuales de cada departamento que permitan alcanzar los objetivos organizacionales.

El modelo propuesto, se enfoca a dos niveles:

1. El Estratégico: enfocado en las operaciones propias de la empresa

2. El Táctico: enfocada en los ejes sustantivos de la empresa: planeación, programación y control de operaciones (77-78).

A estos niveles, se ha adicionado uno de importancia preponderante como es la información y comunicación, que coadyuva en la difusión e integración de las áreas y procesos empresariales; los procesos internos operan de forma adecuada con miras a alcanzar objetivos y metas esperadas, siempre y cuando, todos los miembros de la organización están debidamente informados de las decisiones que se han tomado a nivel de la alta gerencia.

Así mismo (Olivos \& Orue, 2015), plantean un modelo logístico teórico que permita a las pequeñas y medianas empresas, afrontar mercados abiertos y globalizados, en los que las exigencias de productos con elevada calidad y servicios integrales son los más apetecidos a nivel local e internacional; es por ello la necesidad de buscar herramientas, estrategias e in-sumos que les permita elevar su nivel de competitividad.

Velásquez (2003) citado por (Olivos et al., 2015); sostienen que por las características propias de las PYMES, un modelo de gestión debe adecuarse a este tipo de empresas, el tipo de recursos tecnológicos con los que cuenta, su estructura organizacional fundamentalmente de tipo familiar, la baja cualificación de la mano de obra, el escaso acceso a recursos financieros, entre otros factores, limitantes los procesos de integración necesarios para alcanzar eficiencia y por ende su sostenibilidad y desarrollo (187). 
Partiendo de este análisis, se han planteado múltiples propuestas de modelos de gestión, pero que son limitados de aplicar a empresas y organizaciones pequeñas, debido a su complejidad y demandas innovadoras y tecnológicas de los mismos; por estos hechos se considera necesario sintetizar algunas propuestas de modelos desarrollado para este tipo de empresas que brevemente se describen en la figura 1.

Tabla 1

Autores y Modelos de Gestión Logísticos Propuestos para Pymes

\begin{tabular}{|c|c|}
\hline AUTORES & $\begin{array}{l}\text { PROPUESTO DE MODELO DE GESTIÓN } \\
\text { PARA PYMES }\end{array}$ \\
\hline (Díaz Gómez et al., 2008) & $\begin{array}{l}\text { Se enfocan en aquellas partes o procesos que se pueden } \\
\text { costear y que se considera que son determinantes en la } \\
\text { cadena de suministros: provisión, producción y, } \\
\text { distribución (p: 186) }\end{array}$ \\
\hline
\end{tabular}

(González, C.; Matínez, J.; Estos autores destacan cinco áreas que son claves en un Malcón C.; Cavazos, 2013) modelo de gestión logística de las pymes: aprovisionamiento, almacenamiento, distribución, costos y servicio al cliente (p: 187)

(Velásquez, 2003)

Propone un modelo integral estructurado en tres ciclos que abarca todo el proceso productivo, pero es una propuesta dispersa que no integra los indicadores que se tienen que cumplir.

(Olivos et al., 2015)

Plantean un modelo teórico que se plantea como una solución integral, se enfoca en cuatro áreas que considera claves para las pymes: Inventarios, almacenamiento, producción $\mathrm{y}$, distribución. El modelo planteado expresa que para tener éxito requiere de un "flujo sincronizado de información" (p:189), en todas las áreas tanto internas como externas, utilizando la estrategia del justo a tiempo. Por ello propone el sistema empujar/jalar: "El sistema de empujar/jalar debe satisfacer al cliente en tiempo, lugar, calidad, cantidad, servicio y con el menor costo posible" (p:190)

\section{Modelos de Gestión Logística}

Las empresas en los momentos actuales se enfrentan a una realidad con cambios vertiginosos de productos, procesos y mercados, lo que obliga a buscar mecanismos o estrategias que atiendan esta realidad compleja e incierta y que, demandan niveles de 
competitividad y productividad que las haga sostenibles y sustentables dentro de mercados mundiales. Es por ello que de manera imperiosa y urgente se busca estrategias que las diferencien y sean exitosas en lo que hacen.

Algunos autores han desarrollado modelos de gestión que se adecuen a la realidad que enfrentan las pequeñas y medianas empresas, mismas que se desenvuelven en circunstancias más complejas y difíciles por sus propias características, que las vuelven más vulnerables al tener que competir con grandes corporaciones multinacionales. Para (Velazqués, 2003), entiende a los modelos como aquello que representan a un fragmento de una realidad concreta, algo complejo e interrelacionado que se considera como referente y que se somete a comprobación aplicando ciertas variaciones, lo que permite un mejor entendimiento de los aspectos que representan un hecho o problema.

Es necesario construir el término de la gestión a partir de acepciones conceptuales como acciones emprendidas para el logro de un objetivo, o una secuencia de actividades que deben emprenderse para alcanzar un propósito previamente establecido; los conocimientos deben ser sistematizados y aplicados a los procesos administrativos de: diagnóstico, diseño, planeación, ejecución y control (Del Rio Vásquez, 2017). Se puede aseverar que la gestión es el proceso administrativo de los recursos de la empresa mediante la delegación de funciones; por ello administración y gestión se asumen como términos relacionados o vinculados que tratan sobre el cumplimiento de funciones administrativas planteadas por Fayol de: planear, organizar, dirigir, evaluar y controlar, como lo plantean Hernández (2013), (Restrepo González, 2000)

Entender a la logística como el mecanismo de articulación óptima de los flujos productivos que comprende las etapas de: adquisición de insumos, desarrollo del proceso productivo y los sistemas de comercialización, acompañados de procesos eficientes de información y comunicación de la organización; permite ofrecer productos de gran calidad necesarios para atender mercados globales y altamente competitivos (Olivos et al., 2015).

La gestión logística para el sector de la PYMES, no puede ser abordado como lo hacen las grandes empresas, por cuanto el acceso a recursos tecnológicos, la capacitación, y las estructuras bajo las cuales operan son distintas; pero no se puede dejar de incorporar en estas empresas procesos logísticos eficientes e innovación en función a sus propias características, debido a que esto les permitirá competir de manera efectiva en esquemas de mercados globalizados (Cravo et al., 2014).

Según el informe publicado por el Consejo de Desempeño Logístico (2018), analiza los datos publicados sobre el rendimiento logístico y sus indicadores por países que el Banco Mundial pública, plantea que los procesos logísticos, constituyen la pieza clave en los procesos de intercambio comercial de las naciones, porque de esto depende en la actualidad que, una economía este inmersa en el contexto económico mundial. Para la determinación de una posición estrategia en los procesos logístico de cada país se analiza el desenvolvimiento de parámetros como: eficiencia en aduanas, infraestructura moderna, tramitología mínima para envíos, procesos de seguimiento, en el comercio, puntualidad 
y rastreo en las entregas. Para el caso ecuatoriano en dicho informe, se evidencia avances en los procesos logísticos en el desempeño comercial, lo que ha permitido que el país pase de ocupar el puesto 74 en el 2016 a ocupar el puesto 62 en el 2018; es importante destacar que, se está poniendo especial atención a estos procesos por parte de las entidades estatales, para lograr una inserción eficiente de la producción nacional en el mercado mundial.

En la actualidad la gestión de la producción no es tomada de manera literal, la producción engloba toma de decisiones en cuanto a capital humanos, recursos, insumos, etc; que permitan que los bienes o servicios generados cumplan especificaciones y estándares predeterminados, tiempos óptimos, calidad y costos adecuados, que los factores productivos: trabajo, capital e insumos, sean aprovechados y mezclados de forma eficiente y efectiva para alcanzar los objetivos y metas planteadas por la empresa. Esto se enmarca dentro de la administración estratégica que significa procesos de acción o selección de recursos y estrategias que le permitan llevar a la organización en el camino adecuado de competitividad y desempeño, para alanzar un mejor posicionamiento dentro del mercado (Thompson et al., 2008).

A partir de los comentado en párrafos anteriores, es necesario conceptualizar y entender a la logística en el proceso administrativo de la empresa; que debe estar orientada a la satisfacción plena del mercado, por ello las actividades que sirven de apoyo al proceso deben propender al manejo integral, efectivo y eficiente de quienes son parte de la cadena de abastecimiento de la organización, desde la decisión de adquirir los insumos necesarios para los procesos, hasta la distribución de los bienes o servicios pasando por todo el proceso de producción, incluyendo en la actualidad servicios posventa (Velazqués, 2003). Esta logística se la nombra como "Logística empresarial", que refiere (Avendaño Cardenas \& Silva Guerra, 2018), como la secuencia de actividades internas que buscan generar productos o servicios diferenciados, competitivos y que le den sostenibilidad a la organización.

Actualmente, el enfoque de la logística no sólo se centra en la gestión óptima de la cadena de suministros y procesos que le permiten atender los requerimientos de los clientes, incorporando un valor agregado y a un costo adecuado; es necesario incorporar otras variables claves por los problemas medioambientales que enfrenta el mundo moderno; organismos como la Organización de la Naciones Unidas (ONU) (2010) citado por (Ramírez Meneses et al., 2016), sostiene que la administración de las cadenas de suministros de las empresas deben incorporar a más de factores que permiten alcanzar una eficiencia económica, también administrar eficientemente los impactos ambientales y sociales, esto representa aplicar procesos de "logística inversa" (p. 52), atender el mercado actual sin afectar a las generaciones futuras.

\section{Modelos de gestión de operaciones para pymes innovadoras}

(Velásquez, 2003) junto a un grupo de investigadores de la Escuela de Administración de Negocios (EAN), desarrollaron un conjunto de herramientas y estrategias importantes para propiciar un cambio de una pymes en estado natural en una pequeñas y medianas 
empresas innovadora; es una propuesta que busca acoplarse a la realidad de estas empresas caracterizadas por incipientes recursos tecnológicos, ineficientes estructuras organizacionales, y aspectos culturales bajo los cuales se administra y gestionan este tipo de organizaciones familiares; para que, considerando esta realidad les permita incursionar en los macro-mercados actuales, ajustándose a una competitividad agresiva innovadora y depredadora.

Los procesos de globalización en los cuales se desenvuelven las empresas en los momentos actuales, demanda una constante preocupación por desarrollar estrategias, procesos, insumos y productos; que les permita mantenerse en el mercado y alcanzar una competitividad para enfrentar dichos retos. Esta labor resulta compleja para empresas que se desarrollan con políticas proteccionistas como el caso ecuatoriano que, habiendo adoptado en los últimos años políticas arancelarios temporales para promover la "producción nacional, la productividad y competitividad sistémica" (Resolucion-0372015-Tequila, n.d.), con el fin fortalecerlas dentro del mercado nacional; muy pocas empresas han logrado incorporar procesos innovadores, técnicos, tecnológicos y de gestión que les permita ser más eficientes y competitivas; la estructura empresarial predominante en el país no les ha permitido aprovechar esta coyuntura.

Los procesos de inserción a mercados sustancialmente competitivos, como lo plantea autores como Chebrough (2005); (Castells \& Valls-Pasola, 2005); entre otros citados por (Alvarado \& Fernández de Soto, 2012), sostienen que por este entorno competitivo, se obliga a las empresas a una mejor eficiencia en sus procesos operativos, así como incorporar productos y servicios de elevado valor agregado e innovador. Los procesos de cooperación que se alcancen con gobierno amigos, permite la interacción de empresas tanto en términos comerciales como de cooperación sobre todo con economías altamente desarrolladas y tecnificadas.

Las empresas pequeñas o grandes se enfrentan a diario a estructuras e interrelaciones competitivas y globalizadoras;(Arca et al., 2011), expresan que ante esta realidad toma cada vez más relevancia en la gestión administrativa el tema de la logística, y con ello aspectos básicos como el manejo de las cadenas de suministros. El espacio donde se desenvuelven las relaciones comerciales, demanda bienes de mejor calidad, mejores servicios, mejores precios, es decir mejores estrategias; por ello empresas como la textil, demandan estructuras organizacionales que les permita ser sostenibles, así como incorporar procesos innovadores que las haga más eficientes. El incorporar esquemas logísticos en las actividades productivas, busca controlar o minimizar la incertidumbre existente, es decir atender de manera efectiva, eficiente y adaptada a los cambios que experimenta el mercado.

Otro aspecto importante son los canales de distribución, el sector textil opta por modelos logísticos avanzados, a través de intermediarios; las empresas que han logrado funcionar dentro de estructuras organizadas, pueden contar con una óptima gestión de toda su cadena de suministros con fuerte contenido innovador en todas sus áreas respondiendo al mercado de forma efectiva, eficiente y elástica, en donde los niveles de competitividad 
no solo se mide entre empresas sino incluso entre las propias cadenas de suministros (Arca et al., 2011).

Generar estrategias de transferencia e incorporación de procesos tecnológicos en áreas susceptibles de tecnificar; generará en las MIPYMES la necesidad de inversión para la capacitación, adquisición de tecnología, mejora de sus procesos y avanzar por esquemas innovadores ya sea del producto, o de procesos, o en la comercialización van a permitir alcanzar una mayor satisfacción del cliente, mejor productividad, rentabilidad y competitividad.

(Ospina et al., 2014), proponen las 5 etapas del proceso de vigilancia tecnológica representada gráficamente en la Fig. 1.

Figura 1

\section{Proceso de vigilancia tecnológica}

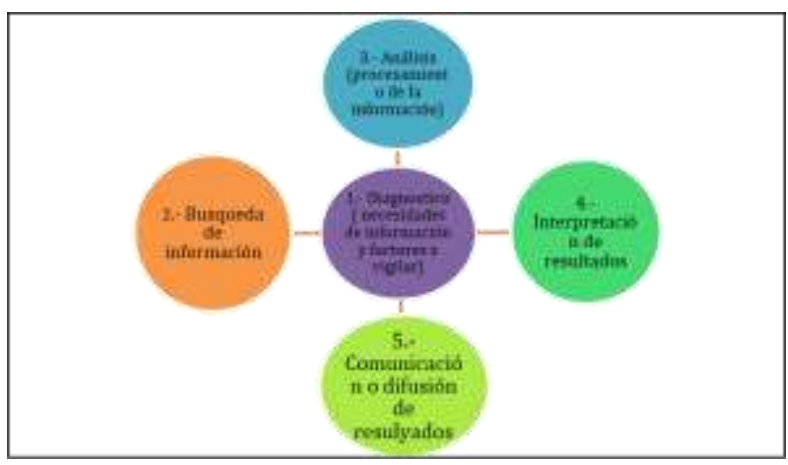

FUENTE: Ospina et. al (2014)

De la revisión bibliográfica seguida por los autores sostienen que las brechas tecnológicas deben ser tratadas y superadas de forma permanente, para no perder competitividad; factor que en otras economías se lo asume de manera permanente lo que permite a la empresa estar posicionada de forma adecuada en los mercados mundiales.

Con el análisis como ejemplo de empresas textiles en Colombia que representan más del $80 \%$ de las ventas de la región, Ospina pudo evidenciar aspectos que se encuentran incorporando las empresas como: componentes de vigilancia tecnológica, mecanismo de información, procesamiento de la información; los autores plantean que los esquemas de vigilancia tecnológica son una herramienta clave para las empresas que quieren mantenerse en el mercado y ser competitivas; por ello, consideran que es importante generar procesos sistemáticos y permanentes de control y seguimiento, si lo que se quiere es contar con áreas y productos que respondan a demandas globalizadas y constantemente renovadas (Ospina et al., 2014).

Castillo (2005) citando por (Larios, 2017), plantea además que el ser humano y sus capacidades son importantes de cultivar para ser competitiva una organización, para alcanzar este objetivo es necesario, promover su formación en áreas administrativasgerenciales que conciban a la tecnología, las comunicaciones y la innovación como 
herramientas básicas de incorporar en los procesos, siempre que se ajusten a su estructura y operatividad de las mismas. Así también Villarán en sus diferentes investigaciones (1998 a , 198b, 2000, 2007) citado por (Larios, 2017), propone que como estrategias se deben promover procesos de cooperación de empresas o sectores ya sea de manera vertical u horizontal y en todas las áreas de las organizaciones; lo que garantizará un eficiente control del ambiente interno y externo y de esta forma asegurar su sostenibilidad en el lago plazo.

Larios (2017) según un estudio diagnóstico del sector textil peruano, establece que el planeamiento estratégico es concebido como una definición de objetivos de corto y mediano plazo, las medianas empresas apenas contaban con una misión y visión, así también evidencia una incipiente capacidad administrativa lo que da cuenta de inexistentes procesos de gestión de calidad, limitando la posibilidad de ser competitivas $\mathrm{y}$ atender efectivamente a mercados mundiales. El trabajo es concluyente al sostener que administrados, gerentes o emprendedores en la actividad diaria se preocupan exclusivamente de las ventas cuantificadas, designando poco o casi nada de tiempo a generar estrategias y la gestión en sí. Otro aspecto que afecta al sector es la falta de generación de productos estandarizados, desarrollados bajo normas técnicas óptimas, lo que repercute en su posición en el mercado, precios, márgenes de rentabilidad y su sostenibilidad tan anhelada. Es necesario que los empresarios se concienticen de la necesidad de capacitación en temas gerenciales, generar acuerdos con los entes gubernamentales y con diferentes instituciones público-privadas tendientes a alcanzar un mejor desarrollo productivo y empresarial; desarrollar una cultura empresarial entendida como el desarrollo de una planificación estratégica participativa; establecer herramientas de capacitación en todo los niveles que permitan obtener productos de adecuada calidad y bajo estándares previamente determinados.

En investigaciones realizada en empresas ecuatoriana del sector textil, (Arguello Mendoza, 2017), ha planteado un modelo de gestión del conocimiento mediante la incorporación de la innovación y el uso de las tecnologías como elemento clave propuesto para la cadena de suministros de las Pymes del sector textiles en la ciudad de Riobamba; entendiendo que es un sector clave para la región y el país en su aporte a la generación de empleo, su función redistributiva de la riqueza y su capacidad de adaptación a los cambios acelerados que demanda el mercado en los tiempos actuales.

\section{Logistica inversa}

Hablar de logística inversa en momentos en que el planeta está seriamente afectado por las ingentes cantidades de desechos que las industrias y los consumidores generan; actualmente las organizaciones que se encuentran enmarcadas en la gestión logística inversa son aquellas que presentan una ventaja competitiva frente a su competencia y porque ahora más que nunca se debe promover una actitud de consumo responsable con el planeta y con las generaciones futuras. Las empresas frente a mercados globales cada vez más tecnificados y mundializados, requiere incorporar procesos logísticos no solo tradicionales, es necesario repensar temas de deterioro del medio ambiente, 
responsabilidad social empresarial, cambios climáticos, niveles de contaminación de fuentes hídricas, etc.; factores que han puesto a las empresas a reestructuras sus cadenas de suministros y procesos productivos, buscando usos alternativos de aquellos bienes que han sido desechados y mediante reprocesos, pueden ser recuperados y generar valor, además de reducir el impacto medioambiental que generan los desechos (Ramírez Meneses et al., 2016).

La logística inversa se ha propuesto como un mecanismo que permite la recolección, proceso - reproceso y comercialización de aquellos bienes que están fuera de uso; partiendo del valor que representa para la organización, la sociedad, el planeta y el medioambiente (Ramírez Meneses et al., 2016). Las Naciones Unidas plantea que las empresas están llamadas a actuar con un mayor compromiso medioambiental dentro de sus actividades productivas, reduciendo los impacto ambientales e incorporando tecnologías amigables con el medioambiente (Ayuso S; Mutis J, 2010).

Entendido lo que representa tener un comportamiento respetuoso con la naturaleza y sabiendo que aquellas empresas que actúan bajo esta óptica están asegurando su permanencia en el mercado; se ha llevado a cabo una revisión minuciosa de literatura sobre los procesos y la incidencia que representa esta logística para muchas empresas, como se detallada en la figura 2 :

Figura 2

Propuestas de implementación de logística inversa

\begin{tabular}{|c|c|c|c|}
\hline NÚMERO & AUTOR & TITULO & PROPUESTA \\
\hline 1 & $\begin{array}{l}\text { (Ramírez } \\
\text { Meneses et } \\
\text { al., 2016) }\end{array}$ & $\begin{array}{l}\text { Aplicación De La } \\
\text { Logística Inversa En La } \\
\text { Administración Eficiente } \\
\text { Del Retorno De Llantas } \\
\text { Fuera De Uso De Las } \\
\text { Empresas De Transporte } \\
\text { De Carga Terrestre En La } \\
\text { Ciudad De Bogotá D.C. }\end{array}$ & $\begin{array}{l}\text { Logística adecuadas para las } \\
\text { actividades de recolección } \\
\text { de productos en desuso, } \\
\text { reproceso o tratamiento y } \\
\text { distribución, } \\
\text { incrementar la eficiencia } \\
\text { operacional de la cadena de } \\
\text { suministros e implementar } \\
\text { políticas de sostenibilidad. }\end{array}$ \\
\hline 2 & $\begin{array}{l}\text { (Rubio } \\
\text { Lacoba \& } \\
\text { Jiménez } \\
\text { Parra, 2016) }\end{array}$ & $\begin{array}{l}\text { La Logística Inversa En } \\
\text { Las Ciudades Del Futuro }\end{array}$ & $\begin{array}{l}\text { Los procesos de gestión de } \\
\text { residuos y de devoluciones } \\
\text { planteado por la logística } \\
\text { inversa para recuperación de } \\
\text { materiales que pueden ser } \\
\text { reutilizados, frente a los } \\
\text { procesos que genera la } \\
\text { logística urbana de } \\
\text { congestión, polución, }\end{array}$ \\
\hline
\end{tabular}


Figura 2

Propuestas de implementación de logística inversa (continuación)

\begin{tabular}{|c|c|c|c|}
\hline NÚMERO & AUTOR & TITULO & PROPUESTA \\
\hline 2 & $\begin{array}{l}\text { (Rubio } \\
\text { Lacoba \& } \\
\text { Jiménez } \\
\text { Parra, 2016) }\end{array}$ & $\begin{array}{l}\text { La Logística Inversa En } \\
\text { Las Ciudades Del Futuro }\end{array}$ & $\begin{array}{l}\text { contaminación entre otros, } \\
\text { que en ocasiones se ven } \\
\text { acentuados los problemas } \\
\text { por políticas caducas. Frente } \\
\text { a estos hechos y al existir } \\
\text { puntos de encuentro y } \\
\text { objetivos comunes entre } \\
\text { estas propuestas se debe } \\
\text { incorporar la logística } \\
\text { inversa a los procesos } \\
\text { logísticos urbanos en bien de } \\
\text { la organización }\end{array}$ \\
\hline 3 & $\begin{array}{l}\text { (Rojas } \\
\text { Conejo, } \\
\text { 2016) }\end{array}$ & $\begin{array}{l}\text { La logística inversa y el } \\
\text { cambio climático }\end{array}$ & $\begin{array}{l}\text { Las empresas ante las graves } \\
\text { afectaciones que ha } \\
\text { generado sus acciones a la } \\
\text { humanidad, debe propender } \\
\text { a un uso eficiente de } \\
\text { recursos mediante la } \\
\text { innovación, proyectos } \\
\text { ecológicos, procesos de } \\
\text { planificación integral de su } \\
\text { actividad productiva e } \\
\text { incorporar reprocesos de } \\
\text { materiales o productos } \\
\text { considerados desechos, esto } \\
\text { permite generar acciones } \\
\text { sostenibles para las } \\
\text { empresas y para el medio } \\
\text { ambiente. }\end{array}$ \\
\hline
\end{tabular}

Sistemas de evaluación de la gestión logística

Supply chain managment $(\mathrm{scm})$

Para saber cómo opera esta propuesta, se parte conceptualizando que SCM hace referencia a un sistema de planificación, la puesta en operación y el seguimiento de los procesos de suministros que estén orientados a la satisfacción del consumidor final. En la actualidad para garantizar la permanencia de las organizaciones en el mercado y ser más competitivas, depende del SCM de la empresa, que le permita alcanzar los menores costos 
del mercado expresados en precios adecuados para su producto, mejor calidad y un sistema operativo flexible y ágil, incorporando procesos tecnológicos modernos. Las empresas sostenibles serán aquella en las que los proveedores son vistos como socios estratégicos que tiene objetivos comunes y aplican procesos logísticos que les provea de eficiencia para administrar su cadena de suministros (Torres, et. al., 2018).

El modelo SCM incorpora algunos elementos que son necesarios de evaluar cómo están siendo administrados por empresas u organizaciones; para el caso de actividades de tipo artesanal cuya característica principal es que se lleva a cabo por personas con un nivel formación básico, con recursos limitados, en zonas rurales con limitado acceso a internet e incipiente desarrollo tecnológico; por ello se han contemplado áreas específicas en las cuales se pueden incorporar herramientas tecnológicas básicas. Por mencionar algunas que pueden ser evaluadas en esta actividad serían: La organización y gestión logística, Técnicas de almacenaje, Sistemas de información, Talento Humano, Barreras del entorno, Medidas de desempeño logístico, logística inversa (Torres, et. al., 2018).

\section{Lean supply chain managment (lscm)}

Este sistema surgió para sistemas de producción en 1990; la palabra "lean", especifica un enfoque de producción diferente concebido como la producción por lotes más pequeños o personalizados con cero desperdicios, que difiere del tradicional o en masa que la mayoría de organizaciones opera bajo este sistema, es decir se habla de procesos de producción más eficientes. El modelo de evaluación de LSCM se plantea para proponer un cuadro de mando, que permita evaluar los resultados de la gestión del LSCM y proveer las herramientas para que los tomadores de decisiones generen acciones operativas eficaces (Rojas Jauregui \& Gisbert Soler, 2017).

La técnica del Lean, también se la conoce como "filosofía esbelta o ágil", se gestiona bajo la filosofía de mejora continua, a través de acciones óptimas en los procesos productivos, o de servicios con el fin de reducir todo tipo de desperdicios y gastos que no aportan valor al producto o proceso; conlleva acciones dinámicas de permanente transformación mediante la implementación de técnicas, instrumentos y mejoras requeridas en todas las etapas del proceso y en las acciones del personal de la organización (Rojas Jauregui \& Gisbert Soler, 2017).

Se dice que esta técnica es de origen japonés expresada en principios que inician con la letra s y se la conoce también como las 5 eses:

- Seiri: Clasificar

- Seiton: Orden

- Seiso: Limpieza

- Seiketsu: Limpieza estandarizada

- Shitsuke: Disciplina (Estevan et al., 2019, p. 4) 


\section{Metodologia.}

Para la construcción de la perspectiva teórica, la presente investigación se enmarca dentro de un diseño bibliográfico de tipo documental a nivel histórico; Hernández Sampiere (2014), argumenta que es el momento de introducirse en el conocimiento que se ha desarrollados dentro de un problema específico y que va a permitir la exposición y el análisis de concepciones y teorías que van a permitir enmarcar a la investigación. En la perspectiva de esta investigación resulta un método válido, el mismo consiste en la contrucción de conceptos a partir de la revisión de literatura relacionada con aspectos referentes a definiciones, modelos de gestión y determinación de indicadores que son determinantes para evaluar los procesos logísticos de la organización, considerando todos aquellos que se han venido proponiendo y aplicando a sectores textiles, principalmente artesanales.

\section{Resultados.}

A partir del presente recorrido teórico, se ha podido determinar:

Que para la implementación de un modelo de gestión logístico para el sector textil artesanal, a más de la logística en la cadena de suministros se deben incorporar los elementos de la logísticas inversa, los graves problemas ambientales que enfrenta la humanidad obliga a las empresas grandes y pequeñas a incorporar esquemas de logística inversa si desean ser competitivas y responsables socialmente.

El liderazgo es un factor clave para la supervivencia de las empresas por el nivel de influencia positiva que genera para el logro de objetivos; por ello contar con una administración preparada en temas sobre: Administrativas-gerenciales que conciban a la tecnología, las comunicaciones y la innovación como herramientas básicas, va a permitir incidir en sus colaboradores para el logro de objetivos y metas planteadas. Se debe tener en cuenta que quien dirige a una organización, debe tener una visión integral del entorno interno y externo que le permita responder de forma oportuna y eficiente a las exigencias de los mercados globales actuales.

Para la construcción de un modelo de gestión logístico para el sector textil artesanal, en base a la teoría desarrollada por (Olivos et al., 2015), se considerará una propuesta integral que atienda a las cuatro áreas claves: Administración de inventarios y proveedores, gestión de almacenamiento, logística para el proceso productive y logística para distribución y mercadeo ajustándose al contexto y características propias de la actividad textil artesanal del cantón Gualaceo- Ecuador.

\section{Conclusiones.}

- Luego de entender la operatividad bajo la cual se desarrolla el sector de las micro, pequeñas y medianas empresas sobre sus procesos actuales operativos $\mathrm{y}$ administrativos que aplica para desarrollar sus actividades, se conoce que los mismos enfrentan escenarios sombríos que les exige, romper paradigmas a partir de entender su funcionamiento, afrontar los nuevos retos del mundo globalizado 
y gestionar conjuntamente con los actores gubernamentales, políticas locales participativas y mundiales que promuevan la actividad reconociendo en las misma su potencial en la generación de empleo, actor clave del desarrollo local sostenible y sustentable y promotor en la mejora de la calidad de vida de la población vulnerable, sabiendo que la misma es parte de la economía popular y solidaria.

- La propuesta del Modelo de Gestión logístico a toda la estructura operativa de una organización, es determinante de los resultados obtenidos en las pymes del sector textil evidenciándose los beneficios del mismo en los indicadores estadísticos de validez, fiabilidad y consistencia. La innovación tecnológica debe incorporarse en todos los procesos para alcanzar mayores beneficios, el conocimiento debe gestionarse desde el conocimiento táctico, es decir el que se adquiere a través de "learning by doing" propuesto por Arrow (1962) es decir se aprende con la acción y; el conocimiento explícito propuesto por Shao, Feng y Lui (2012), que es aquel entendimiento que es perceptible y sistemático, obtenido mediante recopilación de información, analizado y acumulado.

- Existen problemas en el desempeño logístico de la PYMES en América Latina principalmente en lo estructural y muchos más en las empresas pequeñas, se plantean algunas alternativas que permitirá a estas estructuras productivas superar las limitaciones mediante una administración logística es decir: administración y optimización de la cadena de suministros, análisis y construcción de sus propios indicadores, capacitación, innovación tecnológica en la planificación y ejecución logística, fortalecimiento y desarrollo de asociaciones y desde la administración pública a través de: Incentivos para la formación de asociaciones, impulso a procesos de innovación, incentivos para el acceso de las pymes a servicios logísticos, ventanilla única, entre otros.

\section{Referencias bibliográficas.}

Alvarado, R. R., \& Fernández de Soto, J. M. (2012). Estudio de cooperación entre Colombia y Corea del Sur en el sector textil-confecciones. Civilizar, 12(22), 143. https://doi.org/10.22518/16578953.96

Arca, J. G., Prado, C. P., \& Sacaluga, A. M. (2011). El desarrollo de la función logística en la industria alimentaria y textil moda de España. Universia Business Review, $3(31)$.

Arruda, D. O., Mariani, P., Augusto, M., Oliveira, G. De, Castilho, D., \& Augusta, M. (2013). Artesanato com Lã de Ovinos, Turismo e Desenvolvimento Local.

Ashley, C., Roe, D., \& Goodwin, H. (2001). Pro-Poor Tourism Report No . 1 Pro-Poor Tourism Strategies : Making Tourism Work For The Poor A review of experience Caroline Ashley, Dilys Roe and Harold Goodwin. Strategy for Pro-Poor Tourism, 1 .

Avendaño Cardenas, E., \& Silva Guerra, H. (2018). Análisis de los cuellos de botella en la logística internacional de las Pymes de confecciones en Colombia. Telos Revista 
de Estudios Interdisciplinarios En Ciencias Sociales, 20(3), 510-536. https://doi.org/10.36390/telos203.07

Ayuso S; Mutis J. (2010). El Pacto Mundial de las Naciones Unidas - ¿una herramienta para asegurar la responsabilidad global de las empresas? Journal of Globalization, Competitiveness \& Governability / Revista de Globalización, Competitividad y Gobernabilidad / Revista de Globalização, Competitividade e Governabilidade, 4(2), 28-38. https://doi.org/10.3232/GCG.2010.V4.N2.02

Benítez Aranda, S. (2009). La artesanía latinoamericana como factor de desarrollo económico, social y cultural: A la luz de los nuevos conceptos de cultura y desarrollo. Revista de Cultura y Desarrollo, Part 1, 6(Dinámica de la artesanía latinoamericana como factor de desarrollo económico, social y cultural.), 3-19. http://www.lacult.unesco.org/docc/CyD_6.pdf

Castells, P., \& Valls-Pasola, J. (2005). Tecnología e innovación en la empresa.

Competencias, C. N. de. (2013). Plan Estratégico Institucional. Journal of Chemical Information and Modeling, 53(9), 1689-1699.

Consejo Nacional para la igualdad de Género. (2018). Guía Básica PARA LA INCORPORACIÓN DE LA PERSPECTIVA DE GÉNERO E INTERSECCIONALIDAD EN LOS GOBIERNOS AUTÓNOMOS DESCENTRALIZADOS consejo nacional para la igualdad de género Guía Básica PARA LA INCORPORACIÓN DE LA PERSPECTIVA DE GÉNERO E INTERSECCIONALIDAD E.

Cravo, T., Lodato, S., Pires, J., \& Vellani, S. (2014). A Comparative Analysis of IDB Approaches Supporting SMEs: Assessing Results in the Brazilian Manufacturing Sector. Inter-American Development Bank (IDB) Working Paper Series.

Cruz, M. L. R., Manzanares, P. A., García, V. V., \& Ontiveros, M. M. M. (2008). La artesanía como producción cultural susceptible de ser atractivo turístico en Santa Catarina del Monte, Texcoco. Convergencia, 15(46), 225-247.

Del Rio Vásquez, J. L. (2017). Gestión organizacional en entornos complejos por parte de las Mipymes del sector servicios de la ciudad de Sincelejo. Tendencias, 18(2), 45. https://doi.org/10.22267/rtend.171802.75

Delgado, B. (2001). Economía social y economía solidaria: intento de definición. Cayapa. Revista Venezolana de Economía Social, 1(1), 0.

Díaz Gómez, H. B., García Cáceres, R. G., \& Porcell Mancilla, N. (2008). Las Pymes: costos en la cadena de abastecimiento. Revista EAN, 63, 5. https://doi.org/10.21158/01208160.n63.2008.438

Escandon-Barbosa, D. M., \& Hurtado-Ayala, A. (2016). Influence of leadership styles in the performance of Colombian export companies. Estudios Gerenciales, 32(139), 
137-145. https://doi.org/10.1016/j.estger.2016.04.001

Estevan, J., Lucumi, F., Escobar, N. M., \& Sc, M. (2019). Implementación De Justo a Tiempo En El. 1-16.

Fernández, de P. (2015). La valorización artesana y su repercusión turística. El caso de Chile. PASOS Revista de Turismo y Patrimonio Cultural, 13(2), 375-393. https://doi.org/10.25145/j.pasos.2015.13.027

Gazimagomedov, G. (2017). THE ROLE OF FOLK ARTS AND CRAFTS OF DAGESTAN IN CONSOLIDATING AND EXPANDING THE TOURISTEXCURSION ROUTES. South of Russia: Ecology, Development, 12, 161-175. https://doi.org/10.18470/1992-1098-2017-1-161-175

González, C.; Matínez, J.; Malcón C.; Cavazos, J. (2013). METODOLOGÍA DE GESTIÓN LOGÍSTICA PARA EL MEJORAMIENTO DE PEQUEÑAS EMPRESAS. Revista Internacional Administración \& Finanzas, 6(5), 121-130. http://search.ebscohost.com/login.aspx?direct=true\&db=bth\&AN=82259126\&site $=$ ehost-live

Gustami, S. P., Wardani, L. K., Setiawan, A. H., \& Art, C. (2014). Craft Arts and Tourism in Ceramic Art Village of Kasongan in Yogyakarta. Journal of Arts and Humanities, 3(2), 37-49. https://doi.org/10.18533/journal.v3i2.147

Hernández Sampiere, R. (2014). Metodología de la Investigación. Sexta edición (Mc GRaw Hi).

Hill, C. W. L., \& Jones, G. R. (2009). Administración Estratégica. 475.

Jha, G., \& Nath, G. (n.d.). KOTH ( GRASS MAT) CRAFT OF BARUAJANI VILLAGE AS POTENTIAL TOURISM INGREDINT: PROJECT AND DISPLAY Gauhati University Institute of North East India Studies , Guwahati . 1-6.

Katiuska, H. (2013). Propuesta de Guía de Administración Educacional del Ejercicio Jurídico de los estudiantes de Derecho de la Universidad de Autora : Lic . Katiuska Hernández Fraga. Tutor: Dr. Avelino Fernández Peiso . Consultante: MSc. Lourdes de León Lafuente . Consu. 99. http://www.eumed.net/librosgratis/2013a/1311/\#indice

Kirby, Carlos; Brosa, N. (2011). V Foro de Competitividad de las Américas La logística como factor de competitividad de las Pymes en las Américas Autores : Carlos Kirby y Nicolau Brosa La logística como factor de competitividad de las Pymes en las Américas.

Larios, R. P. (2017). Estado actual de las mipymes del sector textil de la confección en Lima. Ingeniería Industrial, 30, 113-137.

Lévesque, B., \& Mendell, M. (2002). L’économie sociale au Québec : éléments 
théoriques et empiriques pour le débat et la recherche. Lien Social et Politiques, 41, 105-118. https://doi.org/10.7202/005149ar

Maruya, K., Yamashita, S., \& Uchiyama, T. (2015). Community spaces in the minds of traditional craftsmen in a pottery village in Japan. Frontiers of Architectural Research, 4(4), 253-262. https://doi.org/10.1016/j.foar.2015.05.002

Massolo, A. (2006). El Desarrollo Local En La Perspectiva De Género. Agricultura, Sociedad y Desarrollo, 3(1), 1-18.

Massolo, A. (2007). El género en el desarrollo local: ¿igualdad de oportunidades entre mujeres y hombres? II ENCUENTRO INTERNACIONAL RETOS DEL DESARROLLO, 2000, 1-17.

Max-Neef, M., Elizalde, A., \& Hopenhayn, M. (2010). Desarrollo a escala humana: $\begin{array}{lllll}\text { opciones para el futuro. Biblioteca } & C F+S, & 150 .\end{array}$ http://www.unibague.edu.co/sitios/ecologia/Desarrollo a escala humana.pdf

Mello, C. I. De, \& Ceretta, C. C. (2015). El Souvenir Artesanal Y La Promoción De La Imagen Del Lugar Turístico. Estudios y Perspectivas En Turismo, 24(2), 188-204.

Olivos, P. C., Carrasco, F. O., Flores, J. L. M., Moreno, Y. M., \& Nava, G. L. (2015). Modelo de gestión logística para pequeñas y medianas empresas en México. Contaduria y Administracion, 60(1), 181-203. https://doi.org/10.1016/S01861042(15)72151-0

Olivos, P. C., \& Orue, F. (2015). Modelo de gestión logística para pequeñas y medianas empresas en México. Contaduría y Administración, 60(1), 181-203. https://doi.org/10.1016/S0186-1042(15)72151-0

Ospina, S., Márquez, C., Juliana, M., López, O., Lucia, O., Mauricio, A., \& Castiblanco, M. O. (2014). Análisis del ciclo de vigilancia tecnológica en las empresas del sector textil del centro sur de Caldas Technology watch cycle Analysis in the textile companies of south central.

Ramírez Meneses, C., Cujar Martínez, D. S., Gutiérrez Mila, I. L., Salazar, O., Barragán, J. G., Rodríguez, J., \& Vanegas, J. L. (2016). Aplicación de la logística inversa en la administración eficiente del retorno de llantas fuera de uso de las empresas de transporte de carga terrestre en la ciudad de Bogotá D.C. Vía Innova, 3, 50. https://doi.org/10.23850/2422068x.473

resolucion-037-2015-tequila.pdf. (n.d.).

Restrepo González, G. (2000). El concepto y alcance de la gestión tecnológica. In Revista Facultad de Ingeniería (Vol. 0, Issue 21, pp. 178-185). http://aprendeenlinea.udea.edu.co/revistas/index.php/ingenieria/article/view/32592 9/20783236 
Richards, G. (2005). TEXTILE TOURISTS IN THE EUROPEAN PERIPHERY: NEW MARKETS FOR DISADVANTAGED AREAS? Tourism Review International, 8, 323-338. https://doi.org/10.3727/154427205774791519

Richards, G., Richards, G., \& Richards, G. (n.d.). Developing and Marketing Crafts Tourism.

Rivera Mateos, M., \& Hernández Rojas, R. D. (2019). Microempresas de artesanía, turismo y estrategias de desarrollo local: retos y oportunidades en una ciudad histórico-patrimonial (Córdoba, España). Estudios Geográficos, 79(285), 529. https://doi.org/10.3989/estgeogr.201820

Rodrigues, B., De Sousa, R., Vasconcelo, V. (2014). Produção artesanal associada ao turismo em ilha grande de Santa Isabel (Parnaíba-Pi). CULTUR: Revista de Cultura e Turismo, 8(2), 137-156.

Rojas Conejo, G. (2016). La logística inversa y el cambio climático. La Logística Inversa y El Cambio Climático, 26(1), 43-48. https://doi.org/10.15517/jte.v26i1.24487

Rojas Jauregui, A. P., \& Gisbert Soler, V. (2017). Lean Manufacturing: Herramienta Para Mejorar La Productividad En Las Empresas. 3C Empresa: Investigación y Pensamiento Crítico, 6(5), 116-124. https://doi.org/10.17993/3cemp.2017.especial.116-124

Rubio Lacoba, S., \& Jiménez Parra, B. (2016). La logística inversa en las ciudades del futuro. Economía Industrial, 400, 69-76.

Sen, A. K. (1998). Las teorías del desarrollo a principios del siglo XXI. Revista Cuadernos De Economía, 29, 73-200. http://econpapers.repec.org/RePEc:col:000093:007577

SRINIVASAN, M.; MUKHERJEE, D.; GAUR, A. (2008). BUYER-SUPPLIER PARTNERSHIP QUALITY AND SUPPLY CHAIN PERFORMANCE: MODERATING ROLE OF RISKS, AND ENVIRONMENTAL UNCERTAINTY. In Grou (Vol. 23529, Issue 2).

Thompson, A. A., Gamble, J. E., \& Peteraf, M. A. (2008). Administracion Estrategica: Mexico Mc Graw-Hill.

Torres, C. Castillo, O. Martínez, K. Flores, L. Calderon, E. (2018). APLICACIÓN DE LA HERRAMIENTA "MODELO REFERENCIAL EN LOGÍSTICA”, PARA CARACTERIZAR LA LOGÍSTICA DE LA EMPRESA HOMECENTER SODIMAC. In Angewandte Chemie International Edition, 6(11), 951-952.

Velasco, J. F. (2015). Acuerdo Ministerial No. DM-2020-063. Ministerio de Cultura y Patrimonio. https://www.gob.ec/sites/default/files/regulations/202108/Documento_Acuerdo_Ministerial_DM-2020-063.pdf 
Velásquez, A. (2003). Modelo de Gestión de operaciones para Pymes innovadoras. Escuela de Administración de Negocios, 7(3), 66-87. https://doi.org/10.1209/epl/1998-00473-7

Velazqués, A. (2003). Available in: http://www.redalyc.org/articulo.oa?id=20604705. Revista Escuela de Administración de Negocios, No.43, 66-87. https://doi.org/01208160

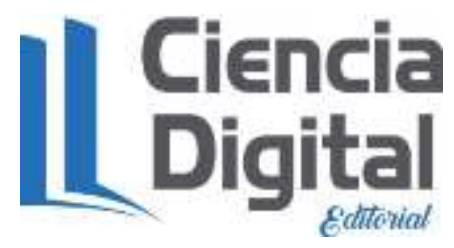




\section{PARA CITAR EL ARTÍCULO INDEXADO.}

Rosales Namicela, M. B. (2021). Revisión teórica de modelos de gestión logístico para el sector artesanal Macanero Gualaceo- Ecuador. ConcienciaDigital, 4(3.2), 19-47. https://doi.org/10.33262/concienciadigital.v4i3.2.1842

\section{\Ciencia}

El artículo que se publica es de exclusiva responsabilidad de los autores y no necesariamente reflejan el pensamiento de la Revista Conciencia Digital.

El artículo queda en propiedad de la revista y, por tanto, su publicación parcial y/o total en otro medio tiene que ser autorizado por el director de la Revista Conciencia Digital.

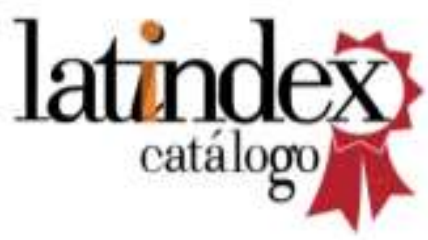

